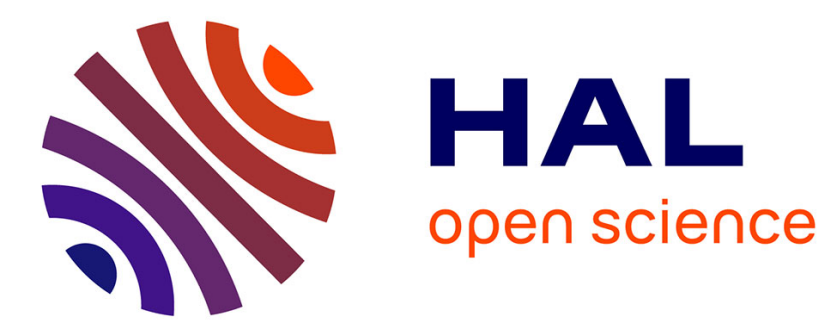

\title{
Insight cognitif et schizophrénie
}

W. El-Hage, N. Lafay, I. Wassouf, N. Jaafari

\section{To cite this version:}

W. El-Hage, N. Lafay, I. Wassouf, N. Jaafari. Insight cognitif et schizophrénie. Annales MédicoPsychologiques, Revue Psychiatrique, 2011, 169 (7), pp.462. 10.1016/j.amp.2011.06.007. hal00789295

\section{HAL Id: hal-00789295 https://hal.science/hal-00789295}

Submitted on 18 Feb 2013

HAL is a multi-disciplinary open access archive for the deposit and dissemination of scientific research documents, whether they are published or not. The documents may come from teaching and research institutions in France or abroad, or from public or private research centers.
L'archive ouverte pluridisciplinaire HAL, est destinée au dépôt et à la diffusion de documents scientifiques de niveau recherche, publiés ou non, émanant des établissements d'enseignement et de recherche français ou étrangers, des laboratoires publics ou privés. 


\section{Accepted Manuscript}

Title: Insight cognitif et schizophrénie

Authors: W. El-Hage, N. Lafay, I. Wassouf, N. Jaafari

PII: $\quad$ S0003-4487(11)00166-1

DOI: $\quad$ doi:10.1016/j.amp.2011.06.007

Reference: $\quad$ AMEPSY 1348

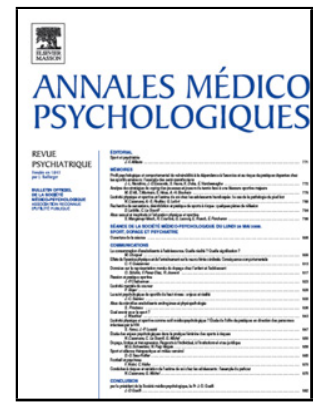

To appear in: Annales Médico-Psychologiques

Please cite this article as: El-Hage W, Lafay N, Wassouf I, Jaafari N, Insight cognitif et schizophrénie, Annales medio-psychologiques (2010), doi:10.1016/j.amp.2011.06.007

This is a PDF file of an unedited manuscript that has been accepted for publication. As a service to our customers we are providing this early version of the manuscript. The manuscript will undergo copyediting, typesetting, and review of the resulting proof before it is published in its final form. Please note that during the production process errors may be discovered which could affect the content, and all legal disclaimers that apply to the journal pertain. 


\title{
Communication affichée
}

\section{Insight cognitif et schizophrénie}

\section{Cognitive insight and schizophrenia}

\author{
W. El-Hage ${ }^{1,2}$, N. Lafay ${ }^{3}$, I. Wassouf ${ }^{3}$, N. Jaafari ${ }^{3}$ Pour Insight Study Group \\ ${ }^{1}$ Clinique Psychiatrique Universitaire, CHRU de Tours, France \\ 2 INSERM U930 ERL CNRS 3106, Université François Rabelais de Tours, France \\ ${ }^{3}$ Unité de Recherche Clinique Intersectorielle CH Henri Laborit, INSERM U 802, CHU \& \\ UFR Médecine et Pharmacie, 86000 Poitiers, France
}

adresse email : n.jaafari@wanadoo.fr

\section{Résumé}

La schizophrénie est souvent associée à une méconnaissance du trouble sévère et persistante. Ce déficit d’insight est corrélé à l'hypofrontalité mais indépendant du pronostic de la maladie ou du quotient intellectuel. L’insight cognitif est défini comme la différence entre la capacité de réflexion sur soi et la certitude dans cette réflexion. Cette capacité est trouvée diminuée dans la schizophrénie mais augmentée en cas de dépression. Ainsi, la schizophrénie avec comorbidité dépressive est associée à un plus haut niveau d’insight. Les auteurs discutent que la prise de conscience de la maladie psychotique peut être vécue comme traumatique, constituant un facteur de risque de dépression et de passage à l'acte suicidaire.

Mots clés : Cognition ; Insight ; Schizophrénie

\begin{abstract}
Schizophrenia is usually associated with severe and chronic lack of knowledge of mental illness. This lack of insight is found to be correlated to hypofrontality but not related to the disorder outcome or to the intelligence quotient. The cognitive insight was defined as the difference between self-reflectiveness and self-certainty. This ability is described as decreased in schizophrenia but increased in depression. Thus, schizophrenia with depressive comorbidity is associated with a higher level of insight. The authors discuss how greater awareness of psychotic illness can be lived as traumatic, which appears to be a risk factor for depression and suicide.
\end{abstract}


Keywords: Cognition; Insight; Schizophrenia

L’insight est un concept composite utilisé essentiellement pour les pathologies psychotiques. Pour certains, le manque d’insight est considéré comme symptôme constitutif de la schizophrénie. Dans la schizophrénie, deux tiers des patients ont une méconnaissance de leur trouble, et jusqu’à $87 \%$ ont une méconnaissance des symptômes spécifiques de leur maladie. L’insight dans la schizophrénie est décrit comme plus faible par rapport à d'autres troubles psychiatriques comme la dépression. Ce déficit sévère et persistant de la connaissance de soi a été mis en lien avec l'hypofrontalité décrite dans la schizophrénie. D’une manière générale, il est admis l'absence de corrélation entre l'insight et le pronostic de la maladie, la sévérité des symptômes, l’observance thérapeutique, le quotient intellectuel, l’âge de début des troubles (Markova \& Berrios, 1995).

Beck et al. (2004) ont développé la notion d’insight cognitif basé sur certains processus cognitifs. L’insight cognitif peut être évalué grâce à la Beck Cognitive Insight Scale (BCIS). Il comprend la capacité de réflexion sur soi et la certitude de soi dans cette réflexion. Chez les patients schizophrènes, la présence d'un délire actif a été trouvé associée à un jugement plus sûr qu'en l'absence de délire actif, avec une corrélation positive modérée entre insight cognitif et dépression. Les patients souffrant d'une schizophrénie sont moins capables de réflexion sur soi que les patients déprimés et les patients déprimés réfléchissent plus sur eux que le reste de la population. Dans la schizophrénie, il a été montré qu’un niveau plus élevé de comorbidité dépressive est associé à un plus haut niveau d’insight, à de meilleures fonctions cognitives et à un plus grand volume cérébral. De même, un plus haut niveau d'insight est associé à un fort risque de suicide chez ces patients. Lors de la dépression postpsychotique, la conscience de la maladie augmente, et la psychose devient la matière de base de la dépression chez ces patients. La prise de conscience de la maladie psychotique est alors comparable à d'autres événements de vie traumatiques, et peut alors être considérée comme un facteur de risque d’une dépression et d’un passage à l'acte suicidaire. 